\title{
Editorial: Neutron Star Physics in the Multi-Messenger Discourse
}

\author{
Gregory Lee Comer* \\ Department of Physics, Saint Louis University, St. Louis, MO, United States
}

Keywords: neutron star (NS), gravitational wave astronomy, fluid dynamic, superfluid, gamma ray: bursts

\section{Editorial on the Research Topic}

\section{Neutron Star Physics in the Multi-Messenger Discourse}

In 2017, Multi-messenger astronomy/astrophysics finally arrived when gravitational waves were directly observed by the LIGO and VIRGO detectors. This event, known as GW170817, triggered a worldwide effort involving 70 observatories on seven continents and in space to gather data across a wide band of electromagnetic radiation (in particular, a short gamma ray burst and kilonova). With little doubt, GW170817 was produced by the inspiral and final merger of two neutron stars (the collapsed cores of regular stars which have exhausted their nuclear fuel). In fact, analysis of the GW170817 remnant contained the tell-tale signs of gold, as well as platinum, confirming nuclear physics predictions that the energetics of neutron star collisions is likely required for natural production of the two elements. This research topic is devoted to the theoretical and computational tools required to model crucial elements of events like that of GW170817.

"Neutron stars (NSs) are extraordinary not only because they are the densest form of matter in the visible Universe but also because they can generate magnetic fields 10 orders of magnitude larger than those currently constructed on earth.” This is a quote taken from this article by Ruiz et al.. They discuss results of a broad physical and computational framework that combines extreme gravity with enormous electromagnetic fields in order to model events like the gravitational wave signal, short $\gamma$ ray burst, and kilonova captured by the detections of GW170817, and the electromagnetic signals GRB 170817 and AT 2017gfo.

In his article Andersson reviews the relativistic multifluid system and how it can cover a wide range of scenarios relevant for different astronomical observation channels: radio and $\mathrm{x}$-ray pulsar timing, gravitational-wave searches, neutron star cooling and associated $\mathrm{x}$-ray observations, longterm evolution of a neutron star's magnetic field, and explosive phenomena like gamma-ray bursts.

In their article Gavassino and Antonelli review recent progress on understanding the mathematical origin of instabilities which occur when non-equilibrium thermodynamics fluid phenomena are considered in relativity.

In their article Krüger et al. review the main results from their recent research on the oscillations of fast rotating neutron stars. They present universal relations (e.g., independent of the equation of state of dense nuclear matter) which combine bulk properties of isolated neutron stars as well as of binary systems before and after merger. Combining these with data on the frequencies of nonaxisymmetric modes of compact objects, they have developed what could become a valuable tool for gravitational wave asteroseismology. 


\section{AUTHOR CONTRIBUTIONS}

The author confirms being the sole contributor of this work and has approved it for publication.

Conflict of Interest: The author declares that the research was conducted in the absence of any commercial or financial relationships that could be construed as a potential conflict of interest.

Publisher's Note: All claims expressed in this article are solely those of the authors and do not necessarily represent those of their affiliated organizations, or those of the publisher, the editors and the reviewers. Any product that may be evaluated in this article, or claim that may be made by its manufacturer, is not guaranteed or endorsed by the publisher.

Copyright (c) 2022 Comer. This is an open-access article distributed under the terms of the Creative Commons Attribution License (CC BY). The use, distribution or reproduction in other forums is permitted, provided the original author(s) and the copyright owner(s) are credited and that the original publication in this journal is cited, in accordance with accepted academic practice. No use, distribution or reproduction is permitted which does not comply with these terms. 